\title{
Magnetic field impact on electrical properties of ZnSe-GaAs solid solutions
}

\author{
A.V. Brodovoy ${ }^{1}$, A.M. Veremenko ${ }^{2}$, V.A. Skryshevsky ${ }^{2}$, A.V. Vlasyuk ${ }^{2}$ \\ ${ }^{1}$ Institute for Problems of Material Science, 3, Krzhyzhanovsky str., Kyiv, Ukraine \\ ${ }^{2}$ Taras Shevchenko Kyiv National University, Radiophysics Department \\ 64,Volodymyrska str.,01033 Kyiv, Ukraine, e-mail:skrysh@univ.kiev.ua
}

\begin{abstract}
The influence of constant magnetic field on thermostimutated current, photoconductivity, and magnetic susceptibility of $\mathrm{ZnSe}-\mathrm{GaAs}$ solid solutions were studied. It was shown that exposition in constant magnetic field results in the concentration decrease of electrically active deep traps. It leads to the change of mechanical and magnetic properties of $\mathrm{ZnSe}-\mathrm{GaAs}$ solid solutions. Proposed was the healing mechanism based on inhomogeneous distribution of the magnetic susceptibility in crystal bulk and, therefore, the appearance of randomly distributed external magnetic fields. Interaction between magnetic moments results in appearance of "driving forces" promoting defect migration and their annihilation.
\end{abstract}

Keywords: solid solution, thermostimutated current, photoconductivity, magnetic field.

Manuscript received 03.10.06; accepted for publication 23.10.06.

\section{Introduction}

It is known that a magnet with the magnetic moment $\vec{M}=\chi \vec{H} \quad$ located in inhomogeneous magnetic field $\vec{H}(z)$ is undergone by the influence of a force $\vec{F}_{r}$ along the direction of the magnetic field gradient. The magnitude of this force $\vec{F}_{r}=M \frac{d H}{d r}$ and direction are determined by the value and sign of a magnetic susceptibility $\chi$ [1]. Appearance of a variable force affecting different parts of the sample with the inhomogeneously distributed magnetic susceptibility $\chi(r)$ in homogeneous magnetic field $\frac{d H}{d r}=0$ can be expected. In it's turn, the inhomogeneous distribution of the magnetic susceptibility can be related to random arrangement of impurities and defects of various origin in the crystal bulk.

Among the examples of these defects, we can note the vacancies, interstitial atoms and their complexes with other impurities, dislocations as well as other combinations of structural imperfections in real crystals. In photosensitive materials, the impurities and structural defects usually serves as recombination centers or nonequilibrium charge capture ones [2]. An information about the force $F_{r}$ and behavior of structural defects can be obtained from investigations of the influence of constant magnetic field on photoelectric processes in crystals. The similar dependence of photoelectric and mechanic properties on magnetic treatment was observed in $\mathrm{Si}$ and GaAs samples [3].

In this work, we study the nonmagnetic solid solutions (SS) of ZnSe-GaAs. Choice of subjects was determined by the high photosensitivity of $(\mathrm{ZnSe})_{1-\mathrm{x}^{-}}$ $(\mathrm{GaAs})_{\mathrm{x}} \mathrm{SS}$. At $x \leq 0.05$, the samples are grown as a large-grained polycrystals. Despite the fit of lattice parameters of initial components to the third decimal place $\left(a_{\mathrm{ZnSe}} \approx a_{\mathrm{GaAs}}=5.653 \AA\right.$ ) [4], the different ionic bond $(\mathrm{ZnSe} \sim 0.7, \mathrm{GaAs} \sim 0.46)$ defines the appearance of the deformation distortions in crystal lattice and high concentrations of various structural imperfections in the bulk of SS, which influences on the magnetic susceptibility and recombination parameters.

\section{Experimental}

Studied were the large-grained polycrystalline $(\mathrm{ZnSe})_{0.95}(\mathrm{GaAs})_{0.05}$ SS samples (a few crystals) obtained using the gas-transport reaction method in chloride and iodide systems. The X-ray structural fluorescent spectral analysis and laser mass-spectroscopy show that the chemical composition of samples is matched up to $x=$ 0.05 with a little excess of Se. 
The optical bandgap of crystals is $E_{g}{ }^{\text {opt }}=2.4 \mathrm{eV}$ (for "pure" $\mathrm{ZnSe} E_{g}{ }^{\mathrm{opt}}=2.7 \mathrm{eV}$ ) [5]. Ohmic contacts are formed at the ends of samples with the dimensions of $0.8 \times 0.4 \times 0.2 \mathrm{~cm}$ using the indium alloying. Dark resistance of crystals at $T=300 \mathrm{~K}$ equals to $10^{7} \ldots 10^{8} \mathrm{Ohm}$, and decreases by one-two orders of magnitude under illumination by scattered light of an incandescent lamp $(50 \mathrm{~W})$. All the samples are of $n$-type with the electron mobility $\mu_{n} \approx 1.8 \cdot 10^{2} \mathrm{~cm}^{2} / \mathrm{V} \cdot \mathrm{s}$ and the concentration $n_{\text {dark }}=10^{9} \mathrm{~cm}^{-3}$.

Photoconductivity relaxation curves of the samples under illumination by light pulses $\left(t \sim 1 \mu \mathrm{s}, h v>E_{g \mathrm{ZnSe}}\right)$ were analyzed. Energy position of capture levels from $c$ band bottom were estimated using the approximation formula $\Delta E=23 k T_{m}$ [6] for the constant heating rate $\beta=0.6 \mathrm{~K} \cdot \mathrm{s}^{-1} ; T_{m}$ is the temperature of maximum of thermostimulated current (TSC) curve $I_{T S C}=f(T)$ for given $\beta$. The capture level concentration $N$ were calculated from the area bounded by the $I_{T S C}=f(T)$ curve. All measurements were performed in the temperature range of 77 to $300 \mathrm{~K}$.

The magnetic susceptibility of SS and its dependence on the magnetic field and temperature were measured using the relative Faraday method. Measurements were performed in the following order. Firstly, we measured the temperature dependence of photoconductivity relaxation curves, thermostimulated current and magnetic susceptibility. Then, the samples were placed between poles of constant magnet and after exposition in the magnetic field of $H_{0}=0.5 \mathrm{~T}$ for 100 hours (further, H-exposition) all the previous measurements were repeated, and these results were compared to the initial data. In each case, the reference samples without H-exposition were used.

\section{Results}

For all the samples before and after exposition in magnetic field, the photocurrent relaxation was fitted by exponential curves with the time constant $\tau$ that depends on a kind of the sample, temperature and exposition time. In accord with the exposition time influence on $\tau$, the samples can be separated into two groups. For the first group, the H-exposition increases $\tau$ without the change of the recombination channel (curves 1, 2 in Fig. 1). The fact that $\tau$ decreases with increasing temperature as $\exp (\Delta E / k T)$ confirms that the electron recombination occurs in the centers with the repulsive Coulomb barrier [6]. The barrier height defined from $\ln \tau \sim \Delta E / k T$ equals to $\Delta E=0.015-0.017 \mathrm{eV}$.

The increase of absolute value $\tau$ (curve $\tau_{2}$ lies over curve $\tau_{1}$ for all $T$ ) during remaining dependence character of $\tau(T)$ shows that concentration of centers with the repulsive Coulomb barrier decreases as a result of magnetic treatment.

For the second group samples, the H-exposition leads to almost complete disappearance of recom-



Fig. 1. Temperature dependences of photoconductivity relaxation time before (1) and after (2) H-exposition for the samples of the first group; $a-$ a possible model of recombination center $\Delta E=0.015 \mathrm{eV}$ (1), $0.017 \mathrm{eV}(2) ; b-$ the plane magnets for $\mathrm{H}$-exposition, 1 - sample.

bination centers with attracting field (Fig. 2, curve 1, model $a$ ) and switch off the recombination channel to negative charged levels with repulsive barrier and height, roughly equal to the first group samples $\Delta E=$ $0.012 \mathrm{eV}$ (Fig. 2, curve 2, model $b$ ). The increase of the absolute $\tau$ value after magnetic treatment evidences that these magnetic field provides the decrease of the recombination center concentration with the repulsive barrier and almost complete disappearance of centers with the attracting barrier.

Influence of H-exposition on the TSC spectrum of these crystals provides a direct evidence of such healing. Fig. 3 shows that TSC curves of initial samples (before $\mathrm{H}$-exposition) consist of three peaks with the following parameters of the energy depth from $c$-band $\Delta E_{c}$ and concentration $N_{i}$ :

$$
\begin{array}{ll}
\Delta E_{1}=0.22 \mathrm{eV} & N_{1}=3.9 \cdot 10^{12} \mathrm{~cm}^{-3} \\
\Delta E_{2}=0.25 \mathrm{eV} & N_{2}=7.3 \cdot 10^{13} \mathrm{~cm}^{-3} \\
\Delta E_{3}=0.48 \mathrm{eV} & N_{3}=1.1 \cdot 10^{14} \mathrm{~cm}^{-3} .
\end{array}
$$

After H-exposition, only the first peak $\Delta E_{1}=0.22 \mathrm{eV}$, $N_{1}=3.9 \cdot 10^{12} \mathrm{~cm}^{-3}$ remains (Fig. 3b). It can be mentioned that the trap levels $\Delta E_{2}$ and $\Delta E_{3}$ disappeared after exposition for 20 hours.

Magnetic field influence on structural defect healing was observed during investigation of TSC in SS crystals with the same composition, but with disturbed surface by supplementary polishing with $14 \mu \mathrm{m}$ grain size abrasive. A distinctive feature of polished crystals, as comparing to the initial ones, consisted in appearance of the high disturbed layer surface conductance, shunting the high resistive bulk of the sample. In the TSC curves inherent to these structures, observed were (Fig. 4a) two peaks with the following parameters

$$
\begin{array}{cc}
\Delta E_{1}=0.28 \mathrm{eV} & N_{1}=2.8 \cdot 10^{12} \mathrm{~cm}^{-3} \\
\Delta E_{2}=0.36 \mathrm{eV} & N_{2}=2.1 \cdot 10^{14} \mathrm{~cm}^{-3} .
\end{array}
$$




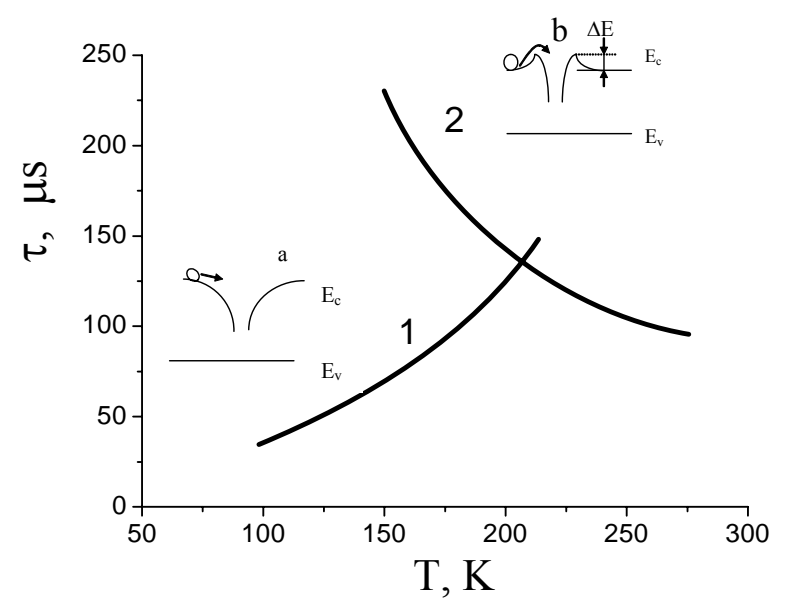

Fig. 2. Temperature dependences of photoconductivity relaxation time before (1) and after (2) H-exposition for the samples of the second group; $a, b$ - possible models of the recombination center for the cases (1) and (2), $\Delta E=0.012 \mathrm{eV}$.

After H-exposition the shunting influence of disturbed layer disappeared, and the initial bulk conductance restored almost completely. In the TSC curve, only the peak with the parameters $\Delta E_{3}=0.31 \mathrm{eV}$, $N_{3}=1.07 \cdot 10^{13} \mathrm{~cm}^{-3}$ is observed.

These listed above results confirm the healing effect of magnetic field on the bulk and surface mechanically created structural defects. This conclusion correlates with the magnetic susceptibility measurement data. Fig. 5a shows the dependences of $\chi$ on the temperature of SS crystals with bulk defects before and after H-exposition. It can be seen that magnetic treatment makes $\chi$ almost one and a half times less. The structural defects created in the surface region during polishing increase the diamagnetic component of $\chi$ (Fig. 5b, curve 3), while the exposition in magnetic field makes $\chi$ two times less (curves 2).

\section{Discussion}

It is known that spins of electrons forming the bond in $\mathrm{A}^{\mathrm{III}} \mathrm{B}^{\mathrm{V}}$ compounds are independent from the kind of the bond (ionic or covalent one) and are always coupled, thus the total spin equals zero. Perfect $\mathrm{A}^{\mathrm{III}} \mathrm{B}^{\mathrm{V}}$ crystals are free from impurities and defects and are always diamagnetic [7]. The susceptibility of a real crystal can be written as a sum of lattice susceptibility $\chi_{\mathrm{A}}$ independent from the temperature and impurity susceptibility $\chi_{\mathrm{S}}(T)$, which essentially depends on the kind and concentration of doping atoms and, in a case of their incomplete ionization, on temperature.

It is evident from the fact that in our case $\chi_{\mathrm{S}}(T)=$ const (Fig. 5), i.e. the lattice defects and other impurities in the temperature range of 77 to $300 \mathrm{~K}$ are almost all ionized. It can be seen from the comparison of curves 1, 2 of Fig. 5a and curves 2, 3 of Fig. 5b that the
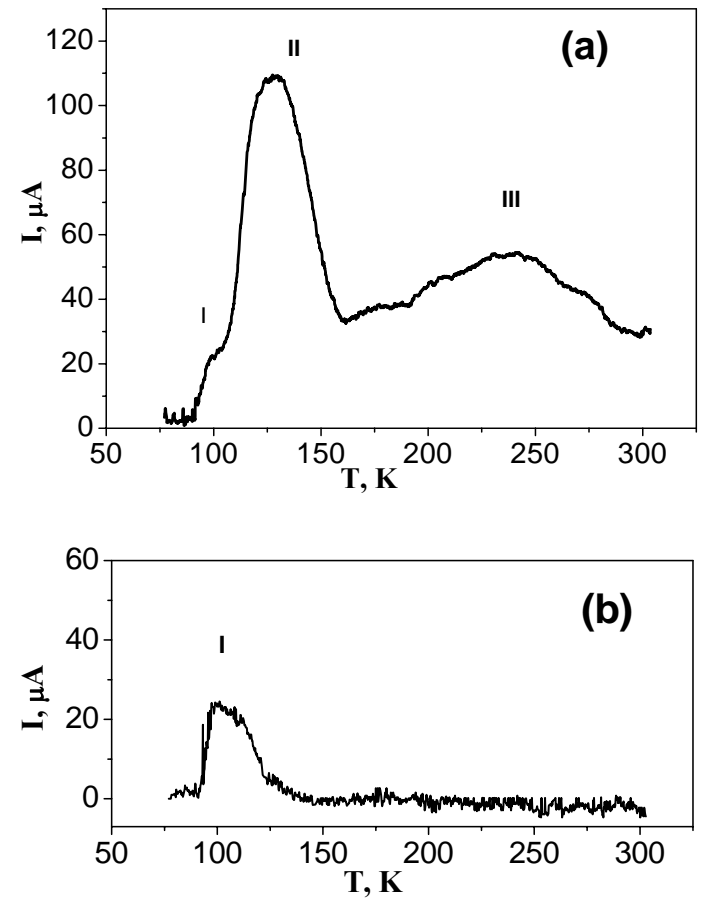

Fig. 3. The TSC curves before (a) and after (b) H-exposition.

total susceptibility $\chi=\chi_{\mathrm{A}}+\chi_{\mathrm{S}}$ decreases at H-exposition, which seemingly is related to defect healing and decreasing the $\chi_{\mathrm{s}}$ component. This healing effect of $\mathrm{H}$ exposition is also confirmed by the influence of magnetic field on TSC curves and photocurrent relaxation.

Crystal lattice disorder during polishing with large grains is accompanied by appearance of significant number of vacancies and interstitial ions in the surface region, which, according to the scheme listed above, causes the growth of total diamagnetism (Fig. 5b, curve 3 ). Since, after H-exposition the photoconductivity increases simultaneously with decreasing diamagnetism, it can be assumed that both effects are related to the disappearance of the same defects. The inhomogeneous distribution of defects and impurities in the bulk leads to the inhomogeneous distribution of magnetic susceptibility. In this case, each part of bulk crystal in external field will be varied by the value of magnetic moment and force appearing between them, which can cause the healing effect.

The most general expression for ponderomotive forces of magnetic field can be obtained from the formula of the magnetic energy $W=\frac{1}{8 \pi} \int \vec{H} \vec{B} d V$. The field energy change $\Delta W$ during arbitrary infinitesimal movement $\vec{r}$ of solids is equal to the work of mechanical forces $\Delta W=\int \vec{r} \vec{f} d V$. The required value of forces $\vec{f}$ can be derived from the obtained $\Delta W$. 

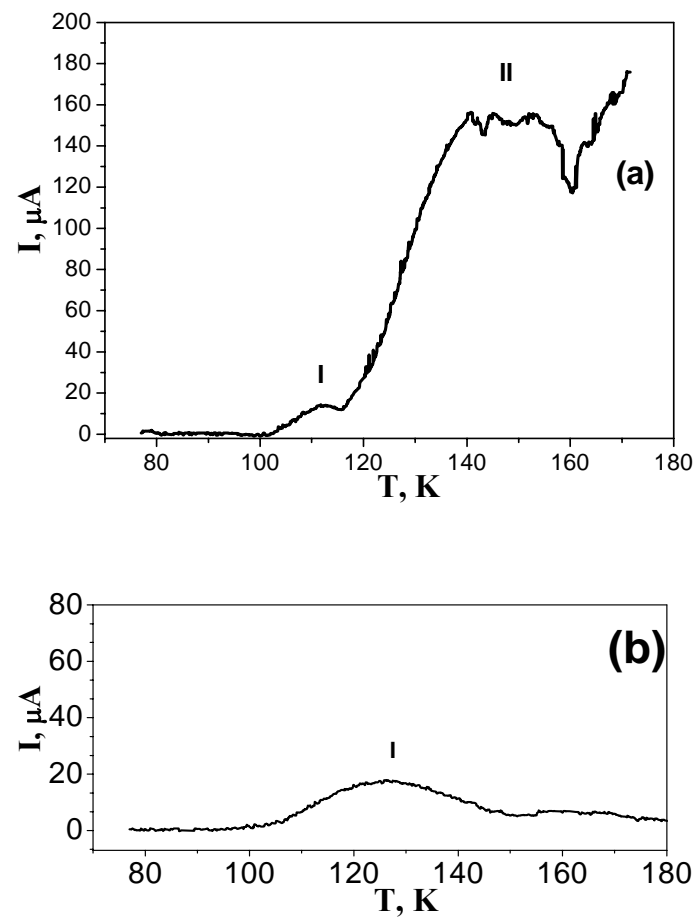

Fig. 4. TSC curve before (a) and after (b) H-exposition for the samples polished with large grain abrasives.
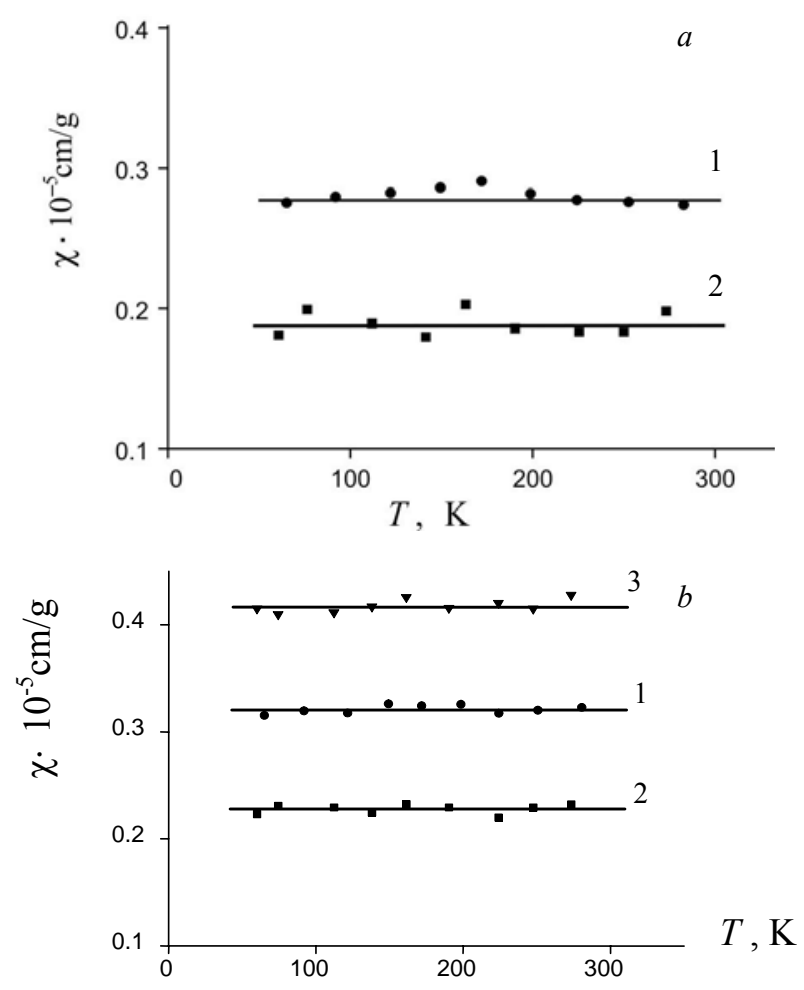

Fig. 5. Dependences of magnetic susceptibility of crystals on temperature, $H=0.3 \mathrm{~T}$ : a) 1 - reference crystals, 2 - after $\mathrm{H}$ exposition of the first group sample; b) 1 - reference crystals, 2 - after $\mathrm{H}$-exposition, 3 - after polishing.
In this model, a similar expression for ponderomotive forces density $f$, i.e., forces affecting the unit of the magnetized volume, according to [8], is equal to

$$
f=f_{1}+f_{2}=\frac{1}{8 \pi} \operatorname{grad}\left(H^{2} \rho \frac{\partial \mu}{\partial \rho}\right)-\frac{1}{8 \pi} H^{2} \operatorname{grad} \mu .
$$

It can be admitted that this expression can be applied only to dia- and paramagnetic environments, when $B=\mu H$. The striction component $f_{1}$ characterizes a dependence of magnetic susceptibility on solid density $\rho$ and manifests itself only in the case of distribution of ponderomotive forces through the bulk of material, while the resulting of the forces applied to all units of volume is equal to zero.

Component $f_{2}$ is equal to the density of ponderomotive forces, affecting the unit of the magnetic volume, placed in magnetic field. Replacing $\mu=1+4 \pi \chi$ and considering the one-dimensional case $\frac{\Delta \mu}{\Delta x}=4 \pi \frac{\Delta \chi}{\Delta x}$, the order of magnitude of $f_{2}=\frac{1}{2} H^{2} \frac{\Delta \chi}{\Delta x}$ can be evaluated. For example, if there is the inhomogeneous distribution of impurities and defects in SS lattice and $\chi$ changes two-three times within the range of one-two lattice distances $\mathrm{ZnSe}\left(a_{\mathrm{ZnSe}} \approx\right.$ $\left.5.6 \cdot 10^{-8} \mathrm{~cm}\right)$, then $\Delta \chi \approx(2-3) \cdot 10^{-6}$ and in the magnetic field $H=5 \cdot 10^{3}$ Oe gives the force density $f_{2}$ $10^{8} \mathrm{dyn} / \mathrm{cm}^{3}$. Dimensionally (energy per volume unit) and physically $H^{2}$ is the force affecting square unit $H^{2} \sim$ $2.5 \cdot 10^{7} \mathrm{dyn} / \mathrm{cm}^{2}$.

It can be said that upon such pressures a change in the SS lattice parameter occurs, the atomic electron shells are deformated essentially, the potential well depth for atoms and ions in interstitials are changed approaching vacancies and interstitials annihilate. If vacancies and interstitial atoms are charged, as it apparently takes place in our case, the additional Coulomb energy makes healing energetically profitable.

\section{Conclusions}

1. By the example of SS crystals of $\mathrm{ZnSe}-\mathrm{GaAs}$, firstly discovered and investigated was a new phenomenon - defect healing in solid states during longterm exposition in the constant, relatively weak $(H=$ $\left.5 \cdot 10^{3} \mathrm{Oe}\right)$ magnetic field.

2. It was shown that such exposition results in decreasing the defect center concentration and changes the electrical and magnetic properties.

3. Proposed was the healing mechanism based on the idea about inhomogeneous distribution of the magnetic susceptibility value in the bulk of crystal and, therefore, appearance of the chaotically distributed magnetic moments in external magnetic field. Interaction between the moments results in appearance of "driving forces" promoting the defect migration, their approachment and annihilation. 


\section{References}

1. L.D. Landau, E.M. Lifshitz, Theoretical physics, vol. VIII, p. 184. Nauka, Moscow, 1982.

2. S.M. Sze, Physics of semiconductor devices. Wiley \& Sons, N.Y., 1981.

3. A.V. Brodovoy, V.A. Brodovoy, V.A. Skryshevsky, Influence of magnetic field on structural effects in $\mathrm{Si}$ and GaAs // Appl. Surf. Sci. 225, p. 170-175 (2004).

4. T. Passow, K. Leonardi, A. Stockmann, H. Selke, H. Heinke and D. Hommel, High-resolution X-ray diffraction investigations of highly mismatched II-
VI quantum wells // J. Phys. D: Appl. Phys. 32, p. A42-A46 (1999).

5. J. Gautron, C. Raisin and P. Lemasson, Optical and electro-optical behaviour of polished and etched zinc selenide single crystals // J. Phys. D: Appl. Phys. 15, p. 153-161(1982).

6. R.H. Bube, Photoconductivity of solids. Wiley, N.Y., 1960.

7. Physics and chemistry of II-VI compounds / Ed. by M. Aven and J.S. Prener. Wiley, N.Y., 1967.

8. I.E. Tamm, Basics of electricity theory. Nauka, Moscow, 1989. 